White surged the foam of the breaking sea around us. We felt our boat lifted and flung forward like a cork in breaking surf. We were in a seething chaos of tortured water; but somehow the boat lived through it, half-full of water, sagging to the dead weight and shuddering under the blow.

Other mariners have been less fortunate. On 12 December 1978, the cargo ship München with 27 people on board sent out a garbled May Day message, and was never heard from again. Pieces of wreckage that were recovered included an un-launched life boat with a pin that had been twisted by an enormous force. The lifeboat was stored $20 \mathrm{~m}$ above the water line. There are many more accounts of extreme waves hitting cruise ships, passenger liners, container ships, tankers, and fishing boats. The fate of the fishing boat Andrea Gail was the basis for the movie The Perfect Storm. The bare statistics are that, over the last 20 years, 200 supertankers and container ships longer than $200 \mathrm{~m}$ have sunk in severe weather, extreme waves certainly being a main suspect. The highest wave ever recorded-quoted in many oceanography textbooks-was the 34-m wave observed in the Pacific by the U.S. Navy-chartered tanker Ramapo in the 1920s. The height was obtained by lining up the wave crest with a line from the top of the foremast to the bridge.

For operationally minded people, any large waves can be called rogues. More precisely, the term rogue is sometimes reserved for waves more than some multiple (perhaps 2 or 2.2) times the significant wave height, defined as the average height of the highest one-third of the

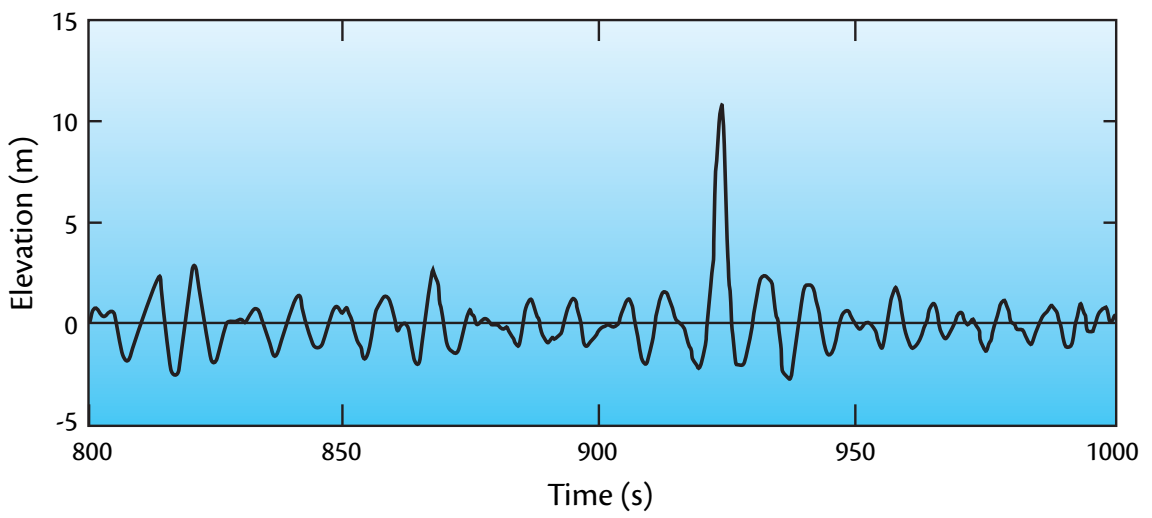

Figure 2. Wave record of November 17, 1984 from the Gorm platform in the central North Sea (Sand et al., 1990). The wave that stands out, though only having a crest height of mere $11 \mathrm{~m}$, clearly exceeds the significant wave height of the background by a factor of two. Another famous wave record is the Draupner wave or "New Year's Wave" where a rogue wave of 25.6-m height was measured in a 10.8-m sea on New Year's Day 1995.

waves. Much of the scientific interest, however, stems from evidence that unusually large waves occur more frequently than would be expected from simple statistics, sometimes appearing within a group of very much smaller waves (Figure 2). More dynamically minded people often reserve the term rogue for these waves that require more than mere statistical superposition. Here we stay with the operational definition.

The quantity of interest depends on the user. For bottom-fixed structures used by the oil industry, it is the crest height that matters, whereas for mariners the height from trough to crest is more important. The direction of propagation is a significant factor; a ship typically heads into large seas and uses its large pitch moment of inertia to limit the response. A ship is vulnerable to large waves coming from a different direction as the roll moment of inertia is much less. This is particularly important if the wave is steep-immense forces can be exerted by a breaking wave on the side of a ship, or on an offshore or coastal structure (Figures 3 and 4 and, for example, Welch et al., 1999).

We would also like to predict the persistence of an extreme wave or the wave group in which it may occur. Can a "wall of water" be spotted enough in advance to allow time for safety measures? Can one identify and track a group within which a rogue wave might suddenly appear? Do extreme waves appear in groups (the "Three Sisters" of mariners' lore)? Are there particular oceanographic conditions in which waves more than twice the significant wave height are more probable than suggested by simple statistics?

Topics covered at the meeting included the basic physics (such as wave-current interactions) that leads to large waves, statistical considerations, and the nonlinear physics that can provide surprises. We 


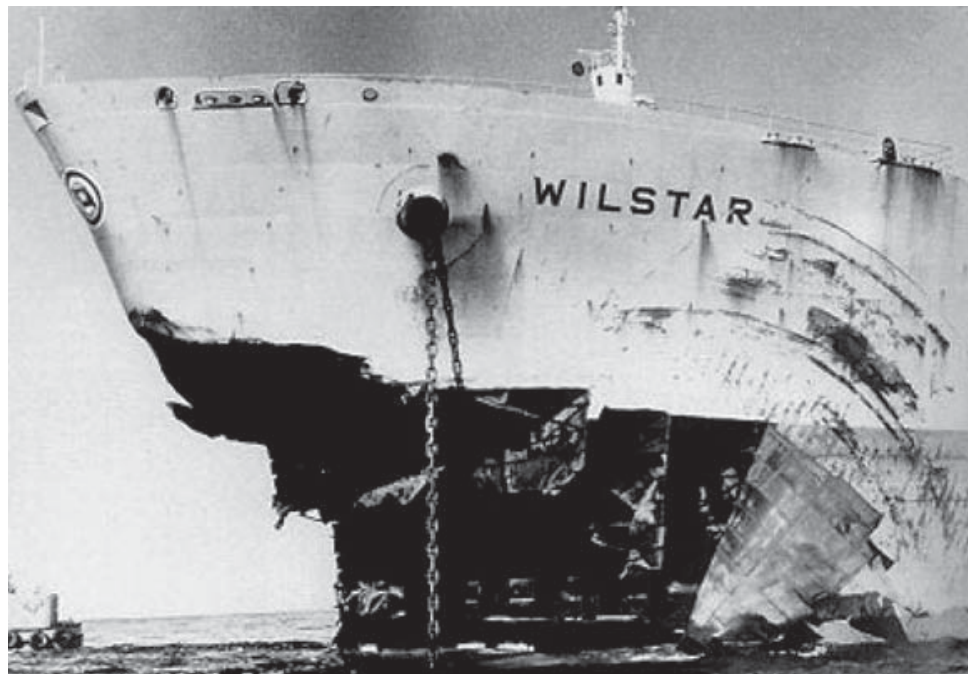

Figure 3. The Wilstar after being hit by a rogue wave in the Agulhas current. To develop standards for the design of ship hulls one must predict not only the likelihood of rogue waves but also the forces that they exert on the hull. Photo credit: DLR.

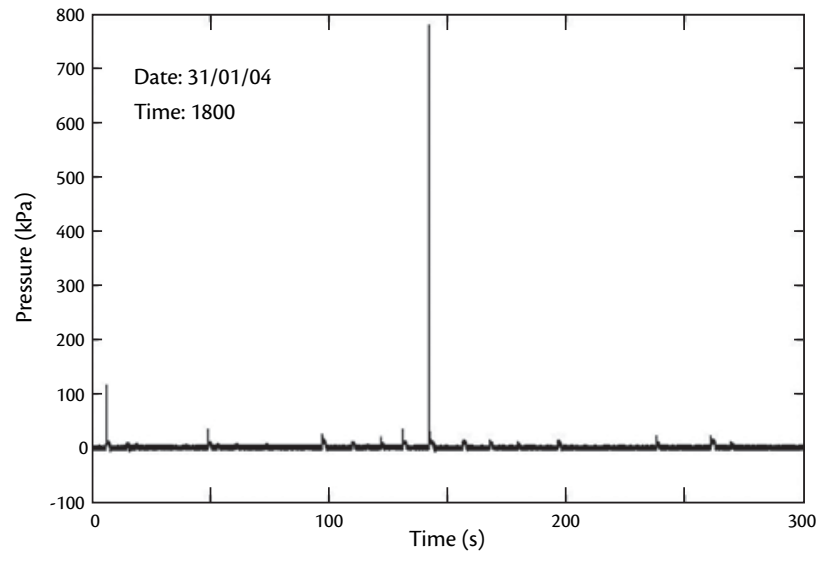

Figure 4. Pressure measurement of an extreme wave recorded at the Admiralty Breakwater, Alderney, Channel Islands, which is exposed to waves from the Atlantic Ocean. The peak pressure is $745 \mathrm{kPa}$, which corresponds to a weight of 74.5 tonnes $/ \mathrm{m}^{2}$. Many ocean-going ships are designed to withstand 15 tonnes/ $\mathrm{m}^{2}$ and may break at 30 tonnes $/ \mathrm{m}^{2}$. Figure courtesy of Howell Peregrine and Charlie Obhrai, University of Bristol, England. also discussed wave breaking as a consequence of large waves and possibly as a limiting factor on their amplitude.

\section{BASIC PHYSICS}

When the wind blows on a calm ocean it first generates short ripples, which are affected by surface tension as well as gravity. Given sufficient time and/or distance offshore, longer waves become dominant. The wave spectrum describes how wave energy is distributed with frequency, and the peak of this spectrum gradually moves to a lower frequency at which waves propagate faster. In a "fully developed" sea, waves at the peak propagate with a speed close to but slightly faster than that of the wind. For a wind speed of $10 \mathrm{~m} / \mathrm{s}$, they propagate with a speed of $11.4 \mathrm{~m} / \mathrm{s}$ and have a period of $7.3 \mathrm{~s}$ and a wavelength of $83 \mathrm{~m}$. It seems that, as the wave spectrum develops, the wind energy is not going directly into the dominant waves present but rather into short waves (Gemmrich et al., 1994). This energy is mostly lost by breaking, and this is how energy (and momentum) is transferred to the current field. The remaining energy is passed by nonlinear interactions to lower frequencies, causing the shift of the peak, and to higher frequencies, where it is dissipated by breaking (Komen et al., 1994).

Various formulae have been fitted to the significant wave height, the period of the dominant waves, and the frequency spectrum that are typically observed for a given wind speed and "fetch" (the distance over which the wind has been blowing). At $10 \mathrm{~m} / \mathrm{s}$ and large fetch, the significant wave height is $2 \mathrm{~m}$, with the wave height defined as the distance from trough to crest. This height increases as the square of the wind speed, so that a sustained $20 \mathrm{~m} / \mathrm{s}$ wind can lead to a significant wave height of $8 \mathrm{~m}$.
Steady winds over large fetches are, of course, rare, so models have been developed to predict wave parameters in general conditions (Komen et al., 1994). The forecasts are very valuable for determining safe and economical routes for merchant shipping. The models allow for energy input by the wind, dissipation by wave breaking, and the interactions that shape the spectrum. In mathematical terms, these interactions rely on phase locking between different waves, but this was traditionally thought to be weak enough that it was reasonable to think of a given sea state as made up of a collection of independent waves that combine in a random manner to give the sea state we observe.

Extra physics that can clearly lead to large waves includes refraction by currents (Figure 5). The simplest example is when waves meet an opposing current; waves with a phase speed $c$ in still water 
can be stopped completely by an opposing current of only $c / 4$. One factor of two is a consequence of surface gravity waves having a group speed (with which energy travels) that is half the phase speed. The other factor of two comes from the reduction in wavelength, and hence in speed, as the waves in front slow down first. If the energy flux is conserved, then the wave height must increase as the group speed decreases. (In fact there is a further increase in wave amplitude because the conserved quantity is actually the flux of "action," or energy divided by intrinsic frequency; the converging current puts more energy into the waves as it squeezes them.) In a sense, the waves are four times more sensitive to opposing currents than one might have expected.

More dramatic increases in wave energy can occur in a two- rather than onedimensional situation. Waves meeting an opposing current jet are slowed more at the center of the jet, focusing energy from the edges. This well-known physics is surely the main reason for the extreme waves frequently encountered in regions such as the Agulhas Current, though there is still more to be understood about the role of fine-scale gradients of the current, its time dependence, and the way in which waves shed their increasing energy via interactions and breaking during their amplification by currents. Moreover, in some locations the gradients of current (or topography in shallow water) are sufficiently sharp that the waves can be diffracted or partially reflected as well as gradually refracted. Although these mechanisms can cause large waves, there is no obvious reason to expect that they will lead to anomalous statistics.

\section{OBSERVATIONS}

Measurements, such as those of Figure 2, made at offshore oil platforms appear to provide a basis for statistical analysis and the identification of unusually

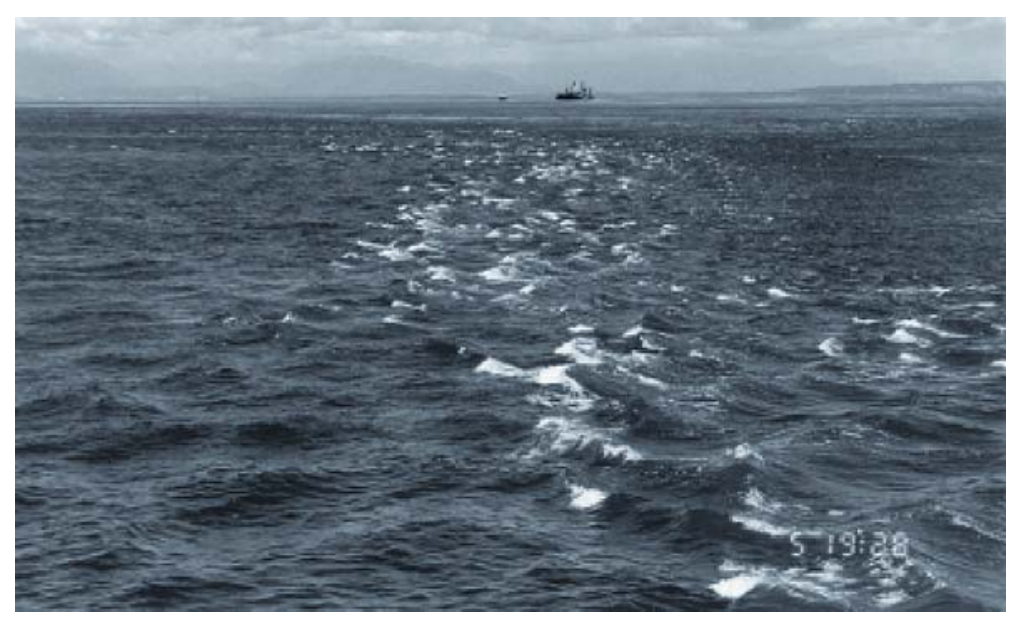

Figure 5. Waves steepening and breaking in an adverse current. This example shows the Stuart Island tidal front in Haro Strait, Canada. It might also represent waves propagating from the Roaring Forties into the Agulhas current when wave heights of tens of meters are imagined. Photo courtesy of Burkard Baschek, Woods Hole Oceanographic Institution. large waves. Unfortunately, even these data can be corrupted by the effects of things like sea spray and the influence of the platform.

Observations of waves from the backscattered signal of short-wavelength radar, either shore- or ship-based, can sometimes provide data on individual waves as well as the average wave height over many wavelengths. Accurate calibration is, however, difficult. The intensity of the backscattered radar signal depends on the slope and amplitude of the scattering wave and the Doppler shift on its orbital velocity. The transformation of these parameters to surface elevation is still a matter of active research. Information on surface wave fields over large areas is also available from space-based synthetic aperture radar (SAR) images (Figure 6), though here too interpretation and translation into wave heights still present challenges.

Altimeter measurements from aircraft are likely to be accurate, but are limited in number.

\section{STATISTICS}

The histogram of wave amplitude (from mean sea level to wave crest) or of wave height (from trough to crest) can be compared with theoretical expectations. More than fifty years ago, Longuet-Higgins (1952) argued that, for a narrowband frequency spectrum, the wave field is a slowly modulated sinusoid with the histogram of wave amplitude being the same as the probability distribution of the wave envelope, and, within the context of linear theory, the wave height is just twice the amplitude. Longuet-Higgins showed that, if the spectrum is made up of many independent com- 

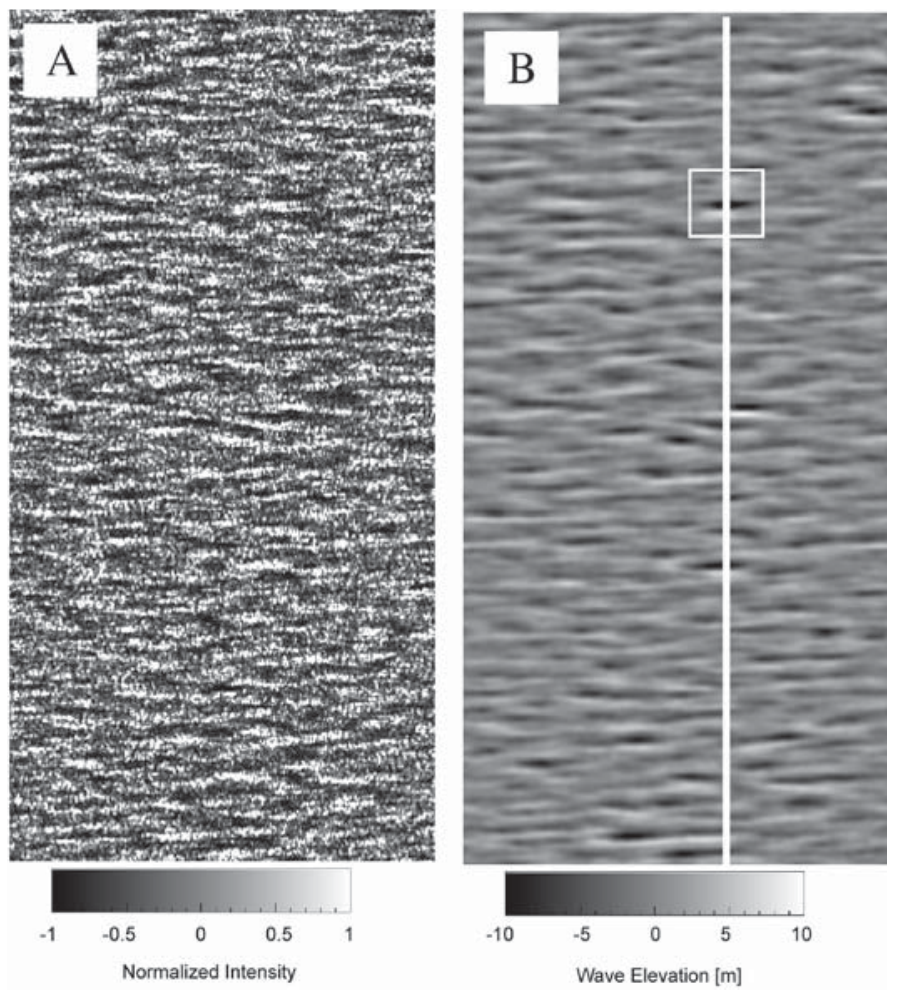

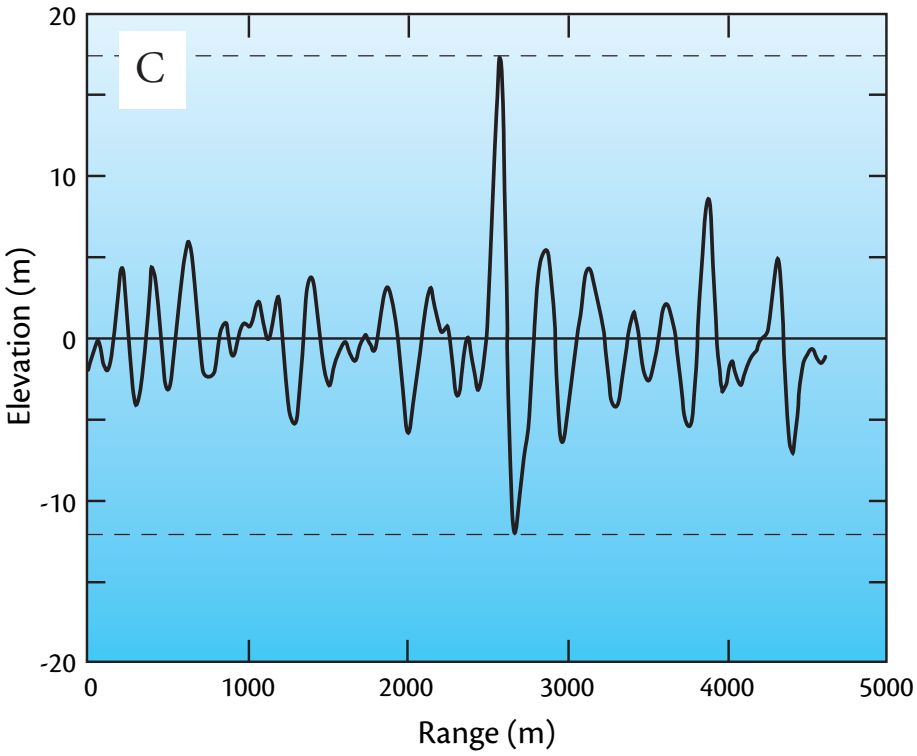

Figure 6. (A) Normalized SAR intensity in a $5 \mathrm{~km} \times 10 \mathrm{~km}$ area at $48.45^{\circ} \mathrm{S}$, $10.33^{\circ}$ E, obtained by ERS-2 on August 27, 1996 at 2244 UTC. (B) Retrieved sea surface elevation, (C) transect along the white line inside the box in $B$. Figure courtesy of Susanne Lehner, University of Miami. ponents, the probability distribution is given by the Rayleigh formula,

$$
P(H)=\frac{H}{4 \sigma^{2}} \exp \left[-\frac{H^{2}}{8 \sigma^{2}}\right]
$$

where $\sigma$ is the rms elevation. (Note that for this probability distribution, the significant wave height $H_{s}$ is equal to $4 \sigma$.)

The tendency of nonlinear waves to have sharper crests than troughs leads to the Tayfun distribution (Tayfun, 1980), which gives a higher probability of rogue waves and which has been found to give an excellent fit to computer simulations of many sea states. A further modification may come from relaxing the requirement for a narrow band spectrum.

There are clearly other major statistical issues that need to be resolved. An anomalously large wave in a short record (such as shown in Figure 2) may not survive tests for statistical significance if it is buried in a longer data set and this includes wave heights from a storm with larger waves in general. On the other hand, as originally remarked by Longuet-Higgins (1952), erroneous conclusions can be reached if a wave record is treated as stationary (i.e., with statistical properties that do not change with time), but is actually non-stationary. An example is shown in Figure 7. Here it is assumed that a wave record is composed of two halves, with the variance in the second half three times that in the first half. The probability of a large wave is considerably greater than would be predicted with the assumption of stationary data. For this example, in fact, one in every 1,300 waves would have $H>2.2 H_{s}$ for the non-stationary situation, as opposed to one in every 16,000 waves if the data were stationary.

There are other difficulties in evaluating what are, after all, typically limited data. Even a time series of wave height at a fixed point really only samples the equivalent of one horizontal dimension, and may not easily be related to the twodimensional statistics of the sea surface. Further, if one is interested in the total wave height, the measurement at a fixed point of the height from a crest to the next trough may differ from what a mariner at the crest would have seen; the trough may shallow before it reaches the fixed sensor! A fixed sensor is also clearly incapable of following the evolution and persistence of an individual wave or group. 
Just gathering reliable data on the occurrence and statistics of extreme waves clearly presents an ongoing challenge. There are also significant difficulties in claiming statistical significance for individual large waves while avoiding burying extreme events in long records. While there are currently no clear answers, there are reasonable grounds for suspecting that more is going on than random superposition.

\section{NONLINEAR PHYSICS}

In the early 1960s, Cambridge fluid dynamicist Brooke Benjamin and his $\mathrm{Ca}$ nadian graduate student Jim Feir found that they could not make a perfectly regular wave train in a simple wave tank. This turned out not to be a consequence of faulty British technology, but rather an unavoidable instability; a single frequency is unstable to sidebands that grow and modulate the wave train. The total energy content is preserved, so some waves must now be larger than the average and so larger than in the expected regular wavetrain.

The Benjamin-Feir instability occurs most readily for waves of a single frequency initially, but a spectrum with a narrow peak will have long groups that resemble a regular wavetrain and so will also tend to break up. The phenomenon has been studied using the full inviscid (Euler) equations in $x$ (along the tank), $z$ (vertical), and $t$ (time) and also with approximate versions in the form of nonlinear equations involving only $x$ and $t$. The models all show a tendency for groups of waves to evolve into sequences of waves that look very like the Gorm event (Figure 2). One model (Janssen, 2003) even gives the probability distribu- tion of large waves in terms of a "Benjamin-Feir Index" evaluated from the narrowness of the spectral peak; the more peaky the spectrum, the longer the basic groups and the more time for the instability to produce extreme waves.

Much of the existing theory is for unidirectional waves. The extension to allow for the directional spreading that is always present is allowed for in some of the model equations and these can still show the emergence of more large waves than would occur without the nonlinear terms in the equations. The model equations are approximate, however, and it is not yet known how directionally spread a spectrum can be and still give rise to anomalous waves. A suspicion is that the answer depends on where the spectral energy is with respect to a two-dimensional instability diagram (Figure 8).

On the (downwind) $\mathrm{x}$ axis, waves close to the primary can interact as in the one-dimensional Benjamin-Feir mechanism, and we see, as discussed earlier, that the interaction will be strongest
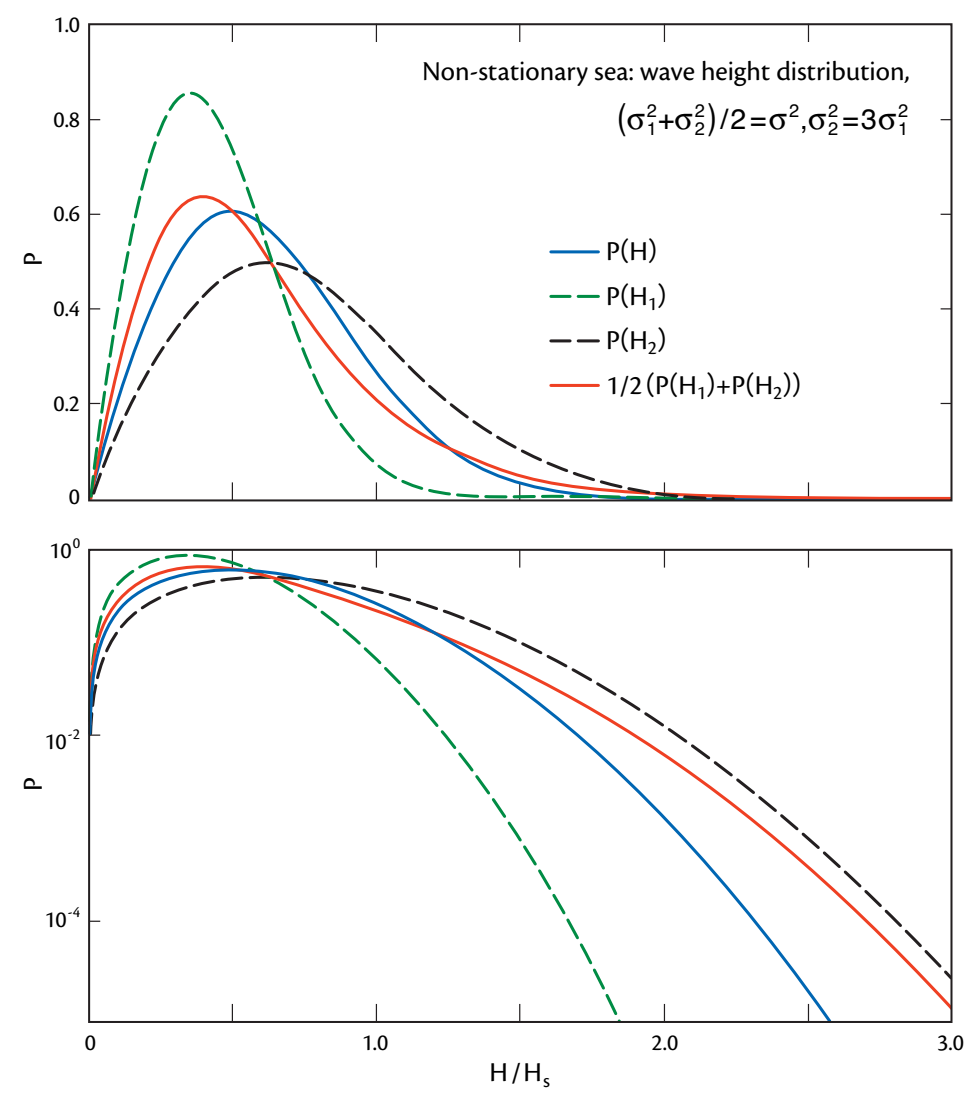

Figure 7. Top: The blue curve shows a Rayleigh distribution of wave height for waves with significant wave height $H_{s}$. The green and black dashed lines show the Rayleigh distributions for records with variance $1 / 2$ and $3 / 2$ that of the average. The red line is the average of these two distributions. Bottom: The same figure with the probability plotted logarithmically. Figure courtesy of Johannes Gemmrich and Chris Garrett, University of Victoria, Canada. 


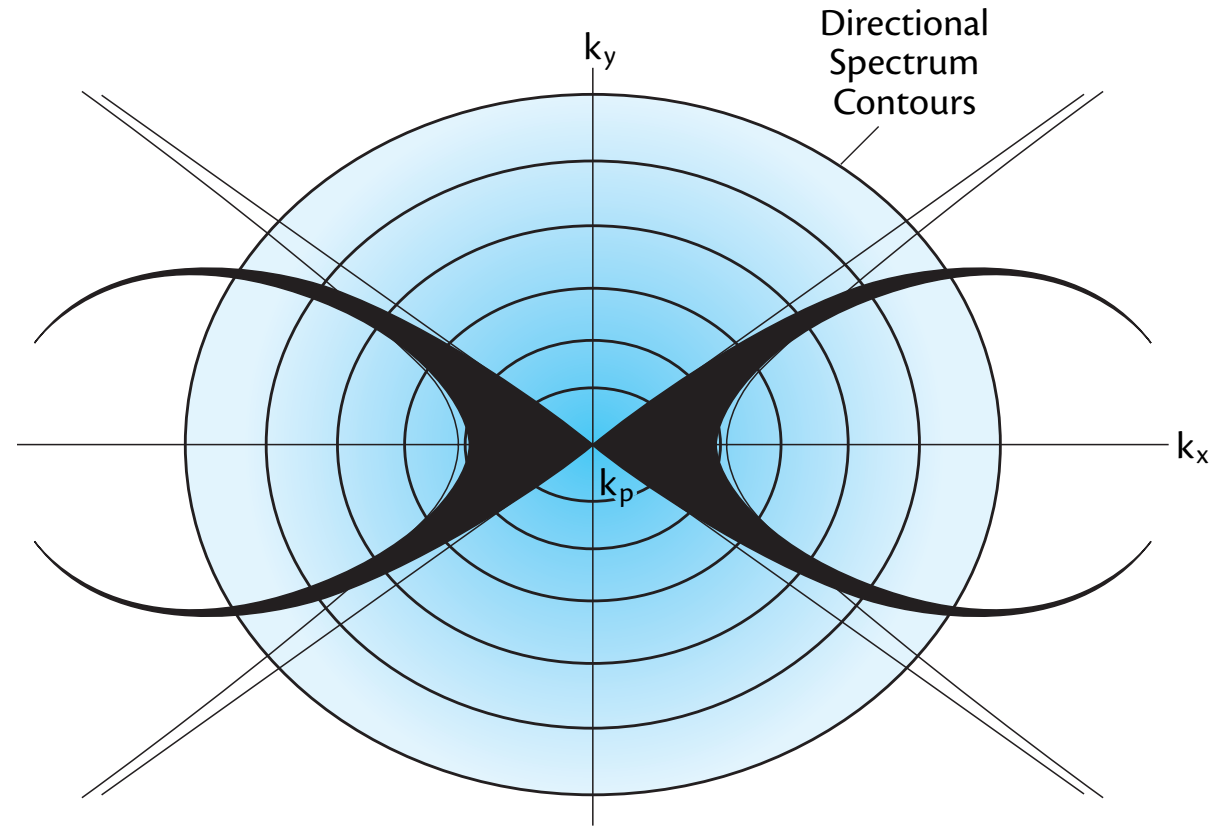

Figure 8 . Wavenumber space, with the origin taken at $\left(k_{p^{\prime}} 0\right)$, the peak wavenumber of a directional spectrum (thin lines) propagating primarily in the $x$-direction. Waves within the black region can interact with $\left(k_{p}, 0\right)$ to produce modulational instability of groups. Figure courtesy of Al Osborne.

if the spectral peak is narrow enough to have most of the wave energy in this region. Off-axis waves are less likely to interact and do so more weakly; waves propagating at more than 35 degrees off the central axis do not contribute at all. It is not yet known how much of the energy of the directional spectrum needs to be within the black region of Figure 8 in order to produce modulational instability and associated rogue waves.

Nonetheless, if further work shows this picture to be correct, it implies that crossing seas may not have anomalous statistics, but may be hazardous just because they can catch ships on their beams. However, Shackleton's observation, and undoubtedly others, may be at odds with this. Perhaps there is something more to be learned about rogue wave formation in crossing seas. We also need further analysis to predict the persistence of individual rogue waves and the groups within which they may spring up.

One way of looking at the BenjaminFeir instability is as a four-wave interaction. A primary wave with frequency $\omega$ enters twice to interact with a sideband frequency $\omega-\delta$ to produce a wave with frequency $2 \omega-(\omega-\delta)=\omega+\delta$. This second sideband interacts in a similar way with the primary to reinforce the first sideband and so on. Of course, for resonance to occur, the wavenumbers must match too, and this is what determines the region of instability.

Resonant nonlinear interactions may occur with larger numbers of waves, though this is unlikely to be important unless the waves are large. In that case, five wave interactions may need to be included, but then wave breaking may be a bigger issue.

As suggestive and exciting as all these theoretical results on the BenjaminFeir instability (and its generalizations) are, it is not yet clear how this mechanism might actually operate in the open ocean. How is a slightly modulated regular wave train set up? Or in spectral terms, how is a narrow band spectrum possible in the first place, since the Benjamin-Feir instability destabilizes and broadens a narrow band spectrum on a time scale faster than, say, the synoptic wind time scale? Establishing favorable initial conditions is certainly a problem.

We have remarked that anomalous statistics are associated with the wavewave interactions causing fixed phase relations between different waves. One type of phase locking that seems strong is between a wave and its first harmonic. This has the familiar consequence that a regular train of surface waves (called Stokes waves) has broad troughs and narrow peaks, as mentioned earlier, with the peaks thus being higher than the troughs are deep. This asymmetry seems to carry over into irregular wave trains (Figure 2). Theoretically, it can be shown that using Stokes waves, rather than sinusoids, as building blocks reduces the degree of nonlinearity in the governing equations.

\section{WAVE BREAKING}

The topic of rogue waves cannot be separated from a discussion of wave breaking. Do rogue waves break and are their heights limited by this? If a rogue wave does break, what are the implications for forces on structures? 
As discussed earlier, wave interactions and wave breaking help to shape the wave spectrum in any conditions. Some rogue waves seem to be a product of strong wave interactions within a narrow spectral peak. There may be similar physics for wave breaking: there are routine breaking events from random superposition of waves of different frequencies, but also possibly anomalous breaking that can arise from the same physics that causes rogue waves.

The standard, and elegant, view of wave breaking (Donelan et al., 1972) started with observations from a commercial flight and is readily confirmed by the reader (though we recommend observations from a few thousand, rather than 35,000, feet).

Consider a group of waves moving across the sea surface. The group moves at half the phase speed of individual crests, so that these crests appear at the rear of the group and propagate through it. A given wave reaches its maximum height at the maximum of the group and may break there. The next wave reaches the same place one period later, but by then the group maximum has moved on half a wavelength. The second wave thus does not catch the group maximum and break there until two periods after the first wave has broken. Thus wave breaking within a group occurs at time intervals of two periods, not one, but at space intervals of one wavelength (Figure 9).

Observations do tend to confirm this, but in long groups there is also a tendency for large breaking waves to emerge by a process called "nonlinear self-focusing" that is essentially the Benjamin-Feir mechanism again. Rogue wave formation and some wave breaking can thus be caused by the same nonlinear physics.

Other factors need to be allowed for. Wave breaking tends to occur for wave steepness $k a>0.3$, at least for unidirectional waves, where $a$ is the wave amplitude and $k$ the wave number equal to $2 \pi$ /wavelength. However, for short fetch situations the wave spectrum that is found already has a steepness close to 0.3 , suggesting that growing waves are inhibited by breaking before they can achieve rogue wave status. This view is supported by observations showing that waves near the peak of the spectrum do indeed tend to break in developing seas, whereas in fully developed seas it is the smaller scale waves that break.

Crossing seas, which occur frequently as the wind direction changes or as the swell arrives from separate storms, may deserve special consideration. As suggested earlier, they may not be prone to the wave-wave interactions that cause anomalous statistics and rogue waves. On the other hand, the development of large waves by superposition may be so rapid for crossing seas that height limitation by breaking is avoided or at least reduced.

\section{THE ROLE OF WIND}

We have been assuming that the role of the wind is just to cause the slow growth of waves, with dynamic rogue wave formation occurring on a shorter time scale and so being unaffected by the wind. Some tank experiments, albeit for unidirectional waves, suggest that this may not be entirely correct.

In particular, the local wind causes a vertical shear in the current; this may stifle the resonant interactions that occur without shear. Some wave breaking is also directly associated with the short waves generated by the local wind. This may interfere with other processes. The role of gustiness in the wind is also far from being fully understood. However, a gusty wind can lead to much larger wave heights. A $20 \mathrm{~m} / \mathrm{s}$ wind with 30\% gustiness (rms deviation), as found often in hurricanes and highly unstable atmospheric conditions, can lead to significant wave heights larger than $10 \mathrm{~m}$, as compared to the $8 \mathrm{~m}$ of a stable wind quoted above (Abdalla and Cavaleri, 2002).

One thing that is clear from wavegeneration models is that the predictions

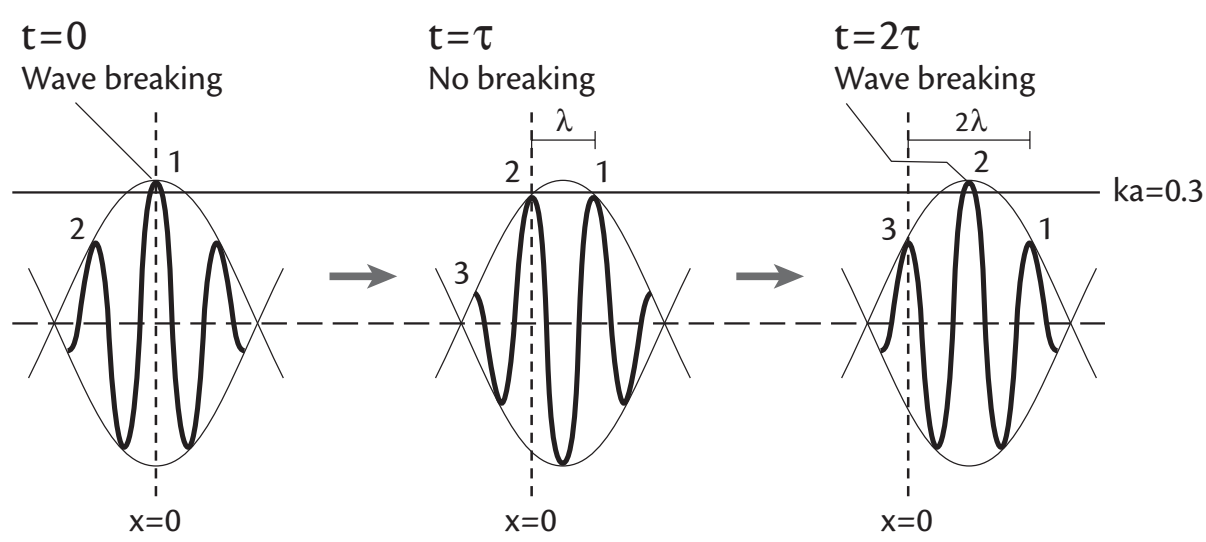

Figure 9. A group of waves with a wave at the peak of the group assumed steep enough to break (taking a critical steepness of 0.3 here). Figure courtesy of Burkard Baschek, Woods Hole Oceanographic Institution. 
are sensitive to variations of wind speed on shorter time and space scales than the $1 \mathrm{~h}$ and $55 \mathrm{~km}$ currently in use on a global scale. Better resolution of the wind is a high priority, particularly in areas with strong spatial and temporal gradients, where presently the models underestimate the peak values for both wind and waves.

\section{WHERE DO WE GO FROM HERE?}

At present, the evidence for dynamical causes of rogue waves is piling up. However, from an objective point of view, because of inaccurate, rare, and biased observations, it is still not easy to completely reject the null hypothesis that rogue waves are due to nothing more than random superposition. Also, onedimensional time series and two-dimensional images of the sea-surface elevation do not allow any direct inferences about its dynamical space-time evolution. often limited to predictions of factors such as the dominant period and significant wave height. An index derived from the narrowness of the spectral peak and its directional spread is flagged in some forecasts as an indicator of possible rogue wave formation (in the sense of a greater than expected occurrence of waves more than twice the significant wave height), but the validity and value of this index needs further study.

Predictions need to be accompanied, of course, by some allowance for the way in which small-scale currents may be increasing the basic wave height.

\section{Theory}

This last point emphasizes that there is a need for more detailed analysis to incorporate wave-current interactions into wave forecast models. The basic physics is understood, but, as described earlier, we do not have a good understanding of the role of fine-scale variations in the cur-

\section{...there is a need for more extensive routine}

measurements with useful precision; at present, too many records of alleged rogue waves

$$
\text { are anecdotal rather than instrumental. }
$$

Nonetheless, a number of operational and research requirements have been hinted at already in this article. We may be more explicit in a number of areas.

\section{Operational Forecasting}

Forecasts of wave conditions used for safety precautions and ship routing are rents, or of the loss of energy from steepened waves by breaking and wave-wave interactions. Many of the existing approaches for deep-water waves also need to be extended to allow for application in shallow water where the seafloor influences wave propagation and interactions.

These theoretical extensions will rely heavily on numerical simulations, most likely using simplified nonlinear model equations that allow for appropriate wave-wave interactions but also include representations of wave breaking and dissipation.

We also include here the need for more sophisticated statistical analyses and appraisal. In particular, questions of stationarity are key to evaluating the frequency of extreme waves.

\section{Wave Tank Experiments}

Controlled experiments in (large) wave tanks have long been used to study the effect of waves on vessels and structures and, more recently, to study the dynamics of highly nonlinear waves. Measurements confirm many predictions of inviscid nonlinear wave theory. The application and relevance of these one-dimensional tank results to the two-dimensional open ocean is a major open research problem that needs to be resolved.

\section{Analysis of Existing Data}

As discussed earlier, the tendency for dynamical rogue wave formation is likely to be very dependent on the directional spread of waves. It would be very useful to have highly resolved directional spectra. These may emerge from real and synthetic aperture data as we learn how to interpret these more accurately.

\section{New Observations}

Our understanding of rogue waves is greatly hampered by the lack of comprehensive observations in space and time. Data at a point cannot provide information on the persistence of individual extreme waves or the environment in which they occur. 
Observational programs in simple situations may be particularly revealing of the basic physics. One possibility is a fetch-limited situation where strong winds blow offshore, though as instrumental. We need to devise instrument packages that can be installed on merchant ships of opportunity, or at least on Navy ships. Data would be particularly valuable from extreme wave

\section{The Marine Observer was a fascinating repository of}

\section{strange observations at sea. Its replacement by a more formal reporting mechanism deserves consideration.}

suggested earlier, in this situation, wave breaking makes the emergence of rogue waves unlikely. Another natural laboratory worth considering is a tidal front, a situation where there are large gradients in currents caused by tidal interaction with topography. In particular, there are estuarine situations where dense water is advected over a sill by a flood tide and sinks below light water, providing a welldefined convergence with dramatic effects on wave height.

In any observational program, techniques can include airborne observations as well as instruments in the ocean. Instruments that can be deployed by air in the path of a hurricane can be a source of unique data. With regards to nearshore programs, for example tidal fronts, radars of different frequency bands will be a particularly valuable tool as they can provide information on currents as well as waves.

Quite apart from focused programs, there is a need for more extensive routine measurements with useful precision; at present, too many records of alleged rogue waves are anecdotal rather than hotspots such as the Agulhas Current.

This is not to discount the value of simple observations by mariners. In particular, the persistence of individual crests is something that might come most convincingly from visual observations; would that Ernest Shackleton had had a stopwatch! The Marine Observer was a fascinating repository of strange observations at sea. Its replacement by a more formal reporting mechanism deserves consideration.

\section{ACKNOWLEDGMENTS}

We thank the participants of the workshop for their input to this report and for their permission to quote unpublished material, and Marshall Tulin for a critical reading of an earlier draft. We also thank Diane Henderson for technical assistance. Copies of the proceedings are available from Peter Müller, University of Hawaii, School of Ocean and Earth Science and Technology, Department of Oceanography, 1000 Pope Road, Honolulu, Hawaii, 96822. The proceedings are available online at $h t t p: / / w w w . s o e s t$. hawaii.edu/PubServices/AhaHulikoa.html.
The fourteenth 'Aha Huliko'a Hawaiian Winter Workshop was supported by the Department of the Navy grant number N00014-00-1-0168, issued by the Office of Naval Research. ⿷匚

\section{REFERENCES}

Abdalla, S., and L. Cavaleri. 2002. Effect of wind variability and variable air density in wave modelling. Journal of Geophysical Research 107(C7/7):1-17, doi 10.1029/2000JC000639.

Donelan, M., M.S. Longuet-Higgins, and J.S. Turner. 1972. Whitecaps. Nature 239:449451.

Gemmrich, J.R., T.D. Mudge, and V.D. Polonichko. 1994. On the energy input from wind to surface waves. Journal of Physical Oceanography 24:2413-2417.

Janssen, P. 2003. Nonlinear four-wave interactions and freak waves. Journal of Physical Oceanography 33:863-884.

Komen, G.J., L. Cavaleri, M. Donelan, K. Hasselmann, S. Hasselmann, and P.A.E.M. Janssen. 1994. Dynamics and Modelling of Ocean Waves. Cambridge University Press, London, UK, 532 pp.

Longuet-Higgins, M.S. 1952. On the statistical distribution of the heights of sea waves. Journal of Marine Research 11:245-262.

Sand, S.E., N.E. Ottesen Hansen, P. Klinting, O.T. Gudmestad, and M.J. Sterndorff. 1990. Freak wave kinematics. Pp. 535-549 in Water Wave Kinematics, A. Tørum and O.T. Gudmestad, eds. Kluwer Academic Publishers, Dordrecht, NL.

Shackleton, E. 1919. South: The Story of Shackleton's Last Expedition 1914-1917. William Heinemann, London, UK, 376 pp.

Tayfun, M.A. 1980. Narrow-band nonlinear sea waves. Journal of Geophysical Research 85:1,548-1,552.

Welch, S., C. Levi, E. Fontaine, and M.P. Tulin. 1999. Experimental study of the ringing of a vertical cylinder in breaking wave groups. International Journal of Offshore Polar Engineering 9:276-282. 